\title{
SOME HOMOTOPY GROUPS OF STIEFEL MANIFOLDS ${ }^{1}$
}

\author{
BY C. S. HOO AND M. E. MAHOWALD
}

Communicated by W. S. Massey, February 1, 1965

Paechter [7] made some computations of $\pi_{k+p}\left(V_{k+m, m}\right)$ where $V_{k+m, m}$ is the Stiefel manifold of $m$ frames in $k+m$ space. In this note we give a table (Table 1) extending his results in the case where $m$ is large. Since $V_{k+m, m} \rightarrow V_{k+m+1, m+1} \rightarrow S^{k+m}$ is a fibering it is clear that $\pi_{k+p}\left(V_{k+m, m}\right)$ depends only on $k$ and $p$ for $p \leqq m-2$. This is called the stable range and we feel that these stable groups are the most important ones. On the other hand for small values of $m$, one of us [4] has made extensive computations and the results are available.

James' periodicity [5, Theorem 3.1 ] is reflected in the table but the basic periodicity of period 8 is also present.

In [1] it is proved that if $n>12$, then $\pi_{j}(S O(n))=\pi_{j}(S O)$ $+\pi_{j+1}\left(V_{2 n, n}\right)$ for $j<2 n-1$. Hence it is easy to deduce the first fourteen nonstable groups of $S O(n)$ from this table.

Tables of homotopy groups are much more useful if generators are given. Instead of generators we settle for giving the order of the image of $i_{*}: \pi_{k+p}\left(S^{k}\right) \rightarrow \pi_{k+p}\left(V_{k+m, m}\right)$ (Table 2). One can construct the generators from this information and this map has important connections with Whitehead products [2].

The groups have been computed by using modified Postnikov towers [6]. An outline of the computation for one case, $6 \bmod 32$, is given. The case $k \equiv 6 \mathrm{mod} 32$. This procedure is essentially the same as the Adams spectral sequence method.

Let $k=32 n+6$ and we suppose $m$ is large. Consider the fibering $V_{32 n+6,7} \rightarrow V_{32 n+m, m+1} \rightarrow V_{32 n+m, m-6}$. We are only interested in groups in the homotopy stable range so that we can construct a new fibering

$$
\Sigma^{-1} V_{32 n+m, m-6} \rightarrow V_{32 n+6,7} \rightarrow V_{32 n+m, m+1} \text {. }
$$

We will build the modified Postnikov tower to this fibering. By [3] the cohomology of $V_{82 n+m, m+1}$ is given by

$$
\begin{aligned}
H^{i}\left(V_{32 n+m, m+1} ; Z_{2}\right) & =0, & 0<i<32 n-1 . \\
& =Z_{2}, & 32 n-1 \leqq i \leqq 32 n+m-1 .
\end{aligned}
$$

Let $h_{i}$ generate $H^{i}\left(V_{82 n+m, m+1} ; Z_{2}\right)$ when it is nonzero. Then $S q^{i} h_{i}$

${ }^{1}$ This research was supported by a grant from the U. S. Army Research Office (Durham). 


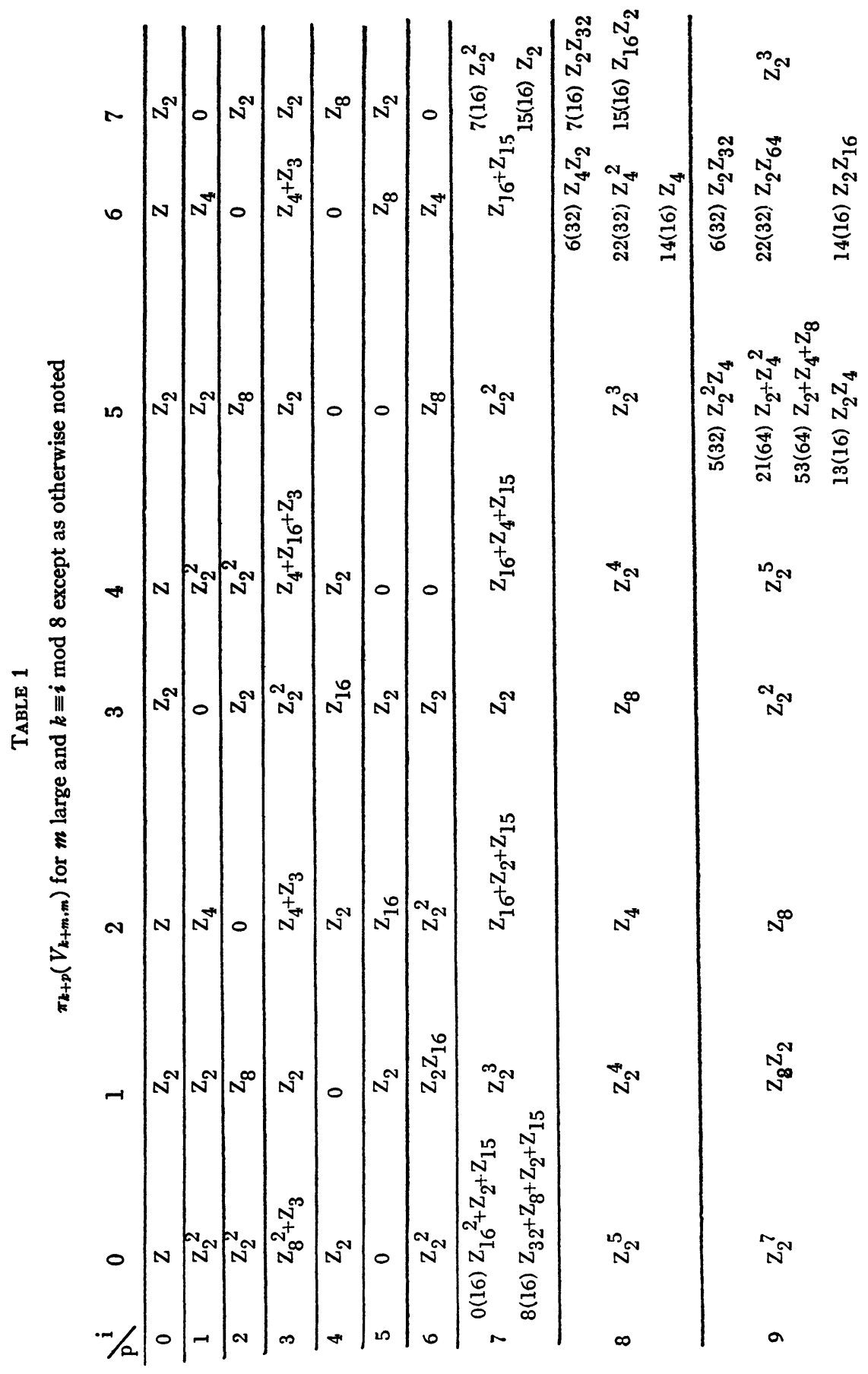




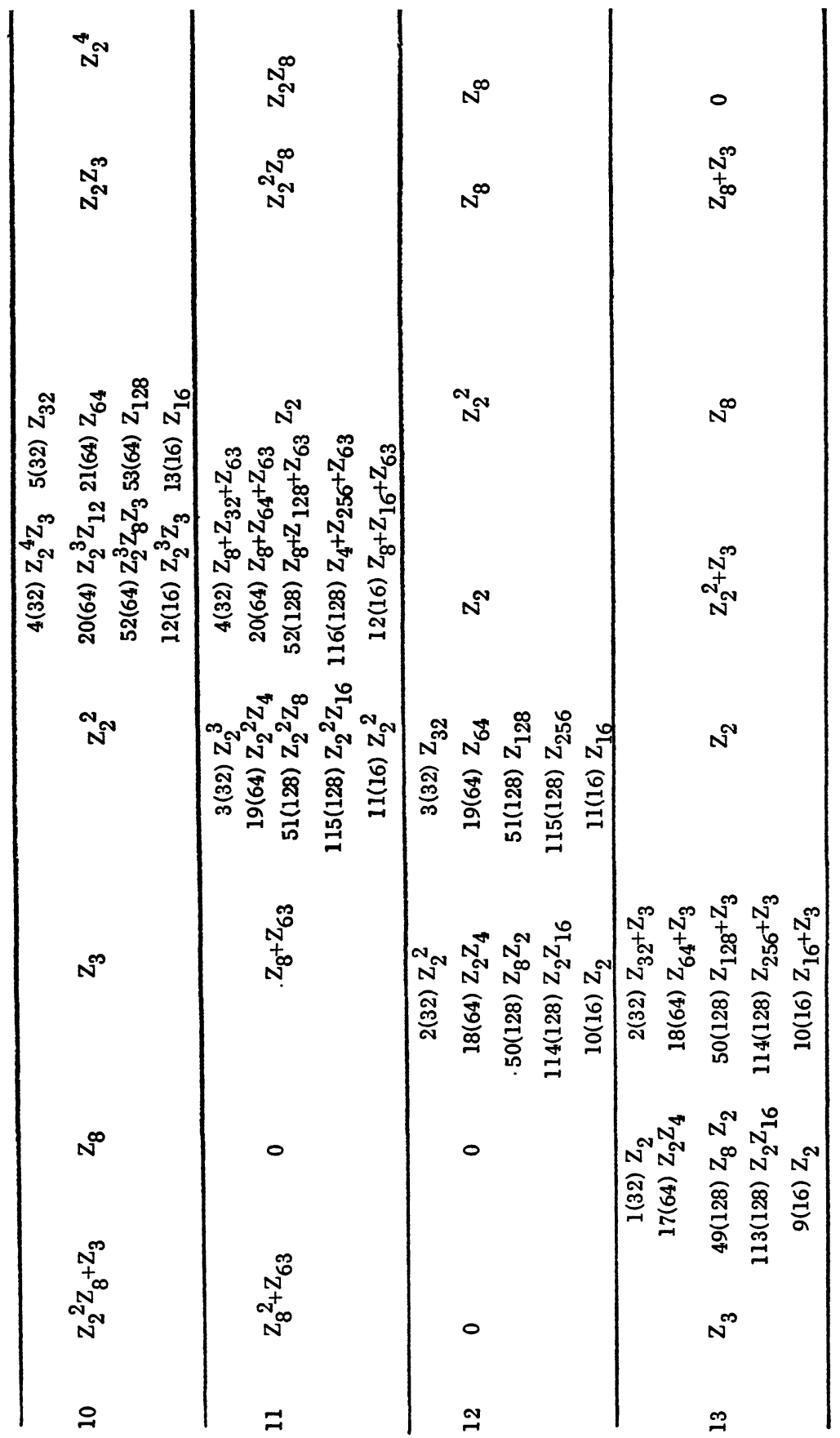


TABLE 2

Order of $\operatorname{im}\left(i_{*} \pi_{j}\left(s^{n}\right) \rightarrow \pi_{j}\left(V_{2 n, n}\right)\right)$

Top row is the name of the stem

\begin{tabular}{ccc|c|c|c|c|c|c|c|c|c|c|c|c|c|c}
\hline \hline$n(8)$ & $\iota$ & $\eta$ & $\eta^{2}$ & $\nu$ & $\nu^{2}$ & $\sigma$ & $\epsilon$ & $\bar{\nu}$ & $\eta \sigma$ & $\eta \epsilon$ & $\eta \bar{\nu}$ & $\eta^{2} \sigma$ & $\mu$ & $\eta \mu$ & $\zeta$ \\
\hline 0 & $\infty$ & 2 & 2 & 8 & 2 & 16 & 2 & 2 & 2 & 2 & 2 & 2 & 2 & 2 & 8 \\
\hline 1 & 2 & 2 & 2 & 2 & 2 & 2 & 2 & 2 & 2 & 0 & 2 & 2 & 2 & 2 & 0 \\
\hline 2 & $\infty$ & 2 & 0 & 4 & 2 & 16 & 0 & 2 & 2 & 0 & 0 & 0 & 2 & 0 & 4 \\
\hline 3 & 2 & 0 & 0 & 2 & 2 & 2 & 0 & 0 & 0 & 0 & 0 & 0 & 0 & 0 & 0 \\
$115(128)$ & & & & & & & & & & & & & & 2 \\
\hline 4 & $\infty$ & 2 & 2 & 8 & 0 & 16 & 2 & 2 & 2 & 2 & 0 & 2 & 2 & 2 & 8 \\
\hline 5 & 2 & 2 & 2 & 2 & 0 & 2 & 2 & 2 & 2 & 0 & 0 & 0 & 2 & 2 & 0 \\
$53(64)$ & & & & & & & & & 2 & & 2 & & & \\
\hline 6 & $\infty$ & 2 & 0 & 4 & 2 & 16 & 2 & 2 & 0 & 0 & 0 & 0 & 2 & 0 & 4 \\
$22(32)$ & & & & & & & & 2 & & & & & & \\
\hline 7 & 2 & 0 & 0 & 0 & 0 & 2 & 0 & 0 & 0 & 0 & 0 & 0 & 0 & 0 & 0 \\
15 & & & & & & 0 & & & & & & & \\
\hline
\end{tabular}

$=(\xi) h_{i+j .}$. Hence the cohomology of the base space is given by $h_{32 n+}$ $=S q^{i+1} h_{32 n-1}$. We let $h_{32 n-1}=h$.

1 st level. Over the Steenrod algebra the basis for ker $p^{*}$ is given by $\left\{S q^{7} h, S q^{8} h, S q^{16} h\right\}$. Of these three only the first two can be spherical in the sense of [6]. Indeed using $i: V_{k+m, m} \rightarrow B S O(k)$ each class in $\pi_{j}\left(V_{k+m, m}\right)$ represents a $k$-plane bundle over $S^{i}$ which becomes trivial when summed with a trivial $m$-plane bundle. It is also easy to see that the bundle is a framed tangent bundle of $S^{j}$ if and only if the cohomology map is nontrivial. Since the $15+32 n$ sphere has only an eight field, $S q^{16} h$ is not spherical. It is useful to kill it anyway but one has to be careful and identify the element at a later stage which is produced because of this.

$2 n d$ level. Consider the following fibering

$$
\begin{aligned}
K_{1}(Z, 32 n+5) \times K_{2}\left(Z_{2}, 32 n+6\right) \times K\left(Z_{2}, 32 n+14\right) & \\
\stackrel{i}{\rightarrow} E^{1} & \stackrel{q}{\rightarrow} V_{32 n+m, m+1}
\end{aligned}
$$

with $k$-invariants $S q^{7} h, S q^{8} h$ and $S q^{16} h$. 


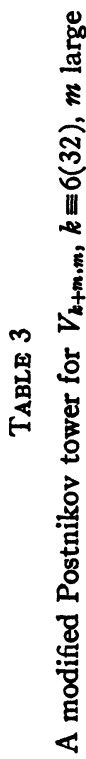

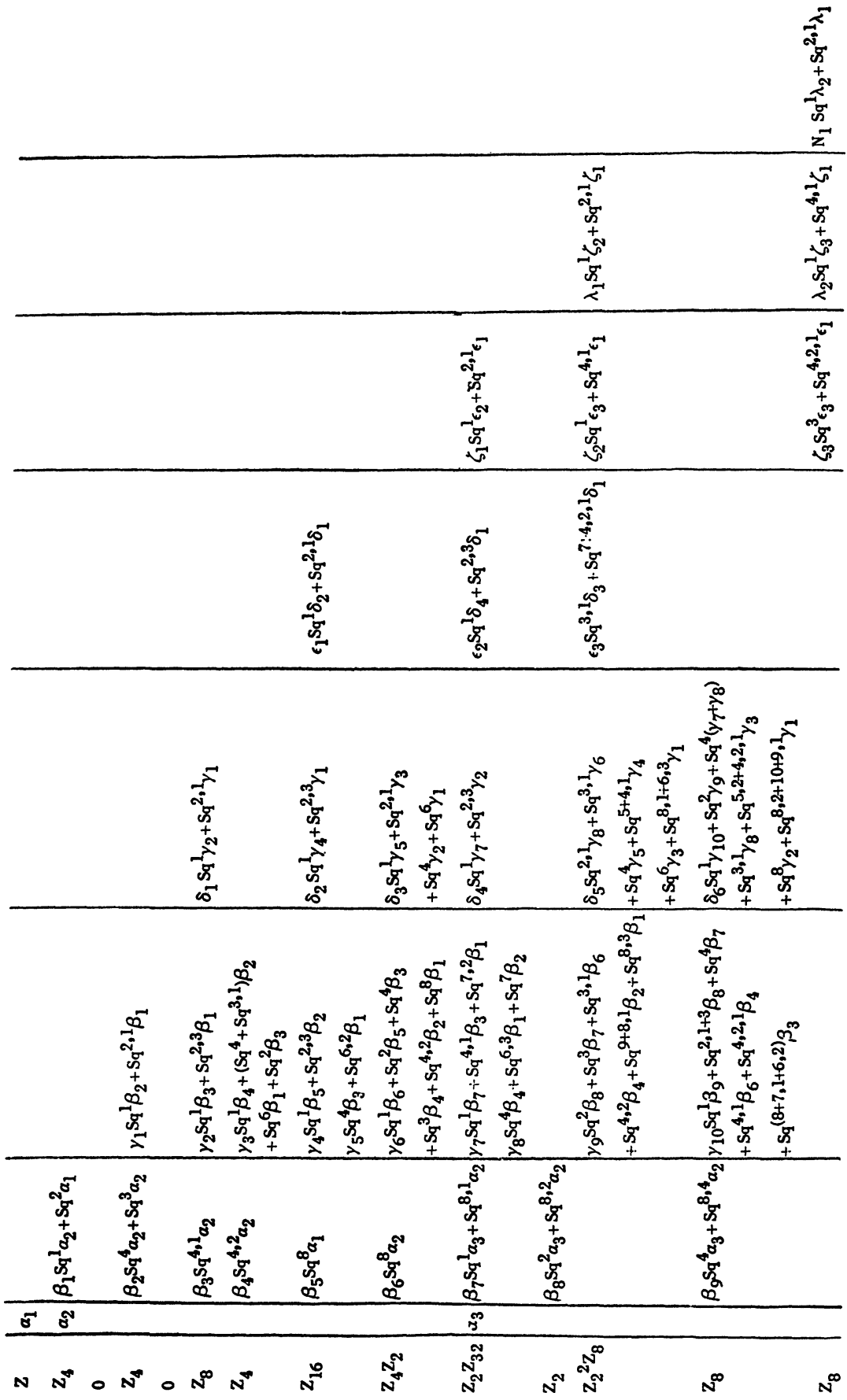


Proposition. $A$ class $\nu \in H^{i}\left(E^{1}, Z_{2}\right)$ such that $\nu \notin \operatorname{im} q^{*}$ and $j \leqq 64 n-3$ satisfies: $i^{*} \nu=\sum_{i=1}^{3} \beta_{i} \alpha_{i}$ where $\alpha_{i}$ is the fundamental class of $K_{i}$ and $\beta_{i}$ is an element of the Steenrod algebra such that $\beta_{1} S q^{7}+\beta_{2} S q^{8}+\beta_{3} S q^{16}$, as an element in the Steenrod algebra, has only classes of length 2 or more in its Cartan basis representation.

Using this representation of $H^{*}\left(E^{1}\right)$ it is now just a lengthy but straight forward computation to verify that the classes in Table 3, column 2 do form a basis over the Steenrod algebra for $H^{j}\left(E^{1}\right)$ if $32 n+7 \leqq j \leqq 32 n+21$.

$3 r d$ level. Consider the fibering

$$
\prod_{i=1}^{9} K_{i}\left(Z_{2}, n_{i}\right) \rightarrow E^{2} \rightarrow E^{1}
$$

with $k$-invariants given by Table 3 . We use $\beta_{i}$ to represent also the fundamental class of $K_{i}$. The value of $n_{i}$ can be inferred from the table. Consider the diagram

$$
\begin{aligned}
& H^{*}\left(E^{2}\right) \stackrel{i_{2}^{*}}{\rightarrow} H^{*}\left(\pi K_{i}\left(Z_{2}, n_{i}\right)\right) \stackrel{\delta^{*}}{\rightarrow} H^{*}\left(E^{2}, \pi K_{i}\right) \\
& \underset{\tau}{\searrow} \underset{H^{*}\left(E^{1}\right) \underset{i_{1}^{*}}{\longrightarrow}}{\simeq} H^{*}\left(K_{1} \times K_{2} \times K_{3}\right)
\end{aligned}
$$

Proposition. $A$ class $\nu \in H^{j}\left(E^{2}\right), 7 \leqq j-32 n \leqq 21$, is defined uniquely by a sum $\sum_{i=1}^{9} a_{i} \beta_{i}$ satisfying:

(1) $i_{2}^{*} \nu=\sum_{i=1}^{9} a_{i} \beta_{i}$ and

(2) $\sum a_{i}\left(i_{1}^{*} \tau \beta_{i}\right)=0$.

This is a special case of 3.3.4 of [6].

Using this proposition the cohomology of $E^{2}$ in the interesting range can be computed. Another lengthy computation shows that column 3 of Table 3 is a basis over the Steenrod algebra for $H^{i}\left(E^{2}\right), 7 \leqq j-32 n$ $\leqq 21$.

4th and higher levels. The computations are made as in the third level, using 3.3.4 of [6]. Nothing unusual happens. The class corresponding to $\gamma_{6}+\delta_{5}$ is the extraneous class produced by killing $S q^{16} h$. This follows from Toda [8]. It is amusing to note that the formula of Adams [0]

$$
S q^{16}=\sum a_{i, j, 3} \phi_{i, j}
$$

with coefficients, for example, $a_{3,3,3}=S q^{1}$ and $a_{1,3,3}=S q^{7}+S q^{4} S q^{2} S q^{1}$, essentially given by $\gamma_{6}$. 


\section{BibliogRAPHY}

0. J. F. Adams, On the non-existence of elements of Hopf invariant one, Ann. of Math. (2) 72 (1960), 20-104.

1. M. G. Barratt and M. E. Mahowald, The metastable homotopy of $O(n)$, Bull. Amer. Math. Soc. 70 (1964), 758-760.

2. - The metastable homotopy of $S^{n}$ (to appear).

3. A. Borel, La cohomologie mod 2 de certains espaces homogenes, Comment. Math. Helv. 27 (1953), 165-197.

4. C. S. Hoo, Homotopy groups of Stiefel manifolds, Ph.D. Thesis, Syracuse University, Syracuse, N. Y., 1964 (mimeographed notes, Northwestern University).

5. I. M. James, Cross-sections of Stiefel manifolds, Proc. London Math. Soc. 8 (1958), 536-547.

6. M. E. Mahowald, Obstruction theory in orientable fiber bundles, Trans. Amer. Math. Soc. 110 (1964), 315-349.

7. G. F. Paechter, The group $\pi_{r}\left(V_{n, m}\right)$. I, Quart. J. Math. Oxford Ser. 7 (1956), 249-268.

8. H. Toda, Vector fields on spheres, Bull. Amer. Math. Soc. 67 (1961), 408-412.

UNIVERSITY OF ILLINOIS AND

NORTHWESTERN UNIVERSITY 